\title{
ON PRE Rg-CONTINUOUS FUNCTIONS IN TOPOLOGY
}

\author{
Govindappa Navalagi ${ }^{1}$ and Sujata Mookanagoudar ${ }^{2 *}$ \\ ${ }^{1}$ Department of Mathematics, KIT Tiptur-572202, Karnataka, India.Email: gnavalagi@ hotmail.com \\ *2Department of Mathematics, Government First Grade College, Haliyal- 581329, Karnataka, India. \\ Email: suja_goudar82@rediffmail.com
}

*Corresponding Author: -

Email: suja_goudar82@rediffmail.com

\begin{abstract}
: -
In this paper we define and study the concept of pre-Rg-continuous functions using preopen sets due to Mashhour et.al (1982) and rg-open sets due to N. Palaniappan (1993). Also,we study the pre-g-regular spaces.

Mathematics Subject Classification (2010): 54A05, 54B05,54C08, 54D10.
\end{abstract}

Keywords: preopen sets, rg-closed sets, gpr-closed sets, rg-continuous functions, rg-irresolute functions.

\section{(ㄷ) (\$) (1)}




\section{INTRODUCTION}

In 1982, A. S. Mashhour et.al [8] has defined and studied the concepts of preopen sets and precontinuous functions in topology. For the first time the concept of generalized closed sets was considered by Levine in 1970 [7]. Later many generalized closed sets have been defined and studied by various Authors in the literature. In 1993, 1997 and 1998 respectively, N. Palaniappan [13], T. Noiri [12] et.al have defined and studied the concepts of rg-closed sets, rg-continuous functions, rg-irresolute functions, gpr-closed sets, gp-closed sets and gp-closed functions in topology. In this paper we define and study the concept of pre-Rg-continuous functions using preopen sets due to Mashhour et.al [8] and rg-open sets due to N. Palaniappan [13].

\section{Preliminaries}

In what follows, spaces $\mathrm{X}$ and $\mathrm{Y}$ are always topological spaces. $\mathrm{Cl}(\mathrm{A})$ and $\operatorname{Int}(\mathrm{A})$ designate the closure and the interior of A which is a subset of $\mathrm{X}$. A subset $\mathrm{A}$ is said to be regular open (resp. regular closed) if $\mathrm{A}=\mathrm{Int}(\mathrm{Cl}(\mathrm{A}))(\mathrm{resp} . \mathrm{A}=\mathrm{Cl}(\mathrm{Int}$ $(\mathrm{A}))$ ).

The following definitions and results are useful in sequel.

Definition 2.1: A subset A of a space $\mathrm{X}$ is called:

(i) Preopen [8] if $A \subset \operatorname{Int}(\operatorname{Cl}(A))$,

(ii) Preclosed [5] if $\operatorname{Cl}(\operatorname{Int}(A)) \subset A$.

The collection of all preopen (resp. preclosed) sets of space X will be denoted by $\mathrm{PO}(\mathrm{X})(\operatorname{resp} . \mathrm{PF}(\mathrm{X}))$.

Definition 2.2: A subset A of $X$ is called preregular [11] if it is both preopen and preclosed set.

The family of all preregular sets of $\mathrm{X}$ is denoted by $\mathrm{PR}(\mathrm{X})$. The complement of a preregular set is also preregular.

Definition 2.3: Let A be a subset of a space $X$ then

i. The intersection of all preclosed sets containing $\mathrm{A}$ is called the pre-closure [5] of $\mathrm{A}$ and is denoted by $p C l(A)$

ii. The intersection of all regular closed sets containing $\mathrm{A}$ is called the r-closure [3] of $\mathrm{A}$ and is denoted by $\mathrm{r} C(\mathrm{~A})$

iii. The union of all preopen sets contained in $\mathrm{A}$ is called the preInterior [9] of $\mathrm{A}$ and is denoted by $\operatorname{pint}(A)$.

iv. The union of all regular open sets contained in $\mathrm{A}$ is called the $r$-Interior[3] of $\mathrm{A}$ and is denoted by $\operatorname{rnt}(\mathrm{A})$

Definition 2.4: A function $\mathrm{f}: \mathrm{X} \rightarrow \mathrm{Y}$ is said to be precontinuous [8] if $f^{-1}(V)$ is preopen in $\mathrm{X}$ for each open set $\mathrm{V}$ of $\mathrm{Y}$.

Definition 2.5: A subset A of a space $X$ is said to be:

i. a generalized closed set (briefly $g$ - closed) [7] if $C l(A) \subset \mathrm{U}$ whenever $A \subseteq U$ and $\mathrm{U}$ is open in $\mathrm{X}$.

ii. a generalized preclosed set (briefly gp - closed) [12] if $\mathrm{p} C l(A) \subset \mathrm{U}$ whenever $A \subseteq U$ and $\mathrm{U}$ is open in $\mathrm{X}$.

iii. a r-generalized closed set (briefly $\mathrm{rg}$ - closed) [13] if $r C l(A) \subset \mathrm{U}$ whenever $A \subseteq U$ and $\mathrm{U}$ is open in $\mathrm{X}$.

iv. a generalized pre-regular closed set (briefly gore - closed ) [6] if $p C l(A) \subset \mathrm{U}$ whenever $A \subseteq U$ and $\mathrm{U}$ is regular open in $\mathrm{X}$.

The complement of a g-closed (resp. gp-closed,rg-closed and gpr-closed) set ofa space X is called g-open (resp. gpopen, rg-open and gpr-open) set in $\mathrm{X}$.

Definition 2.6: A function $\mathrm{f}: \mathrm{X} \rightarrow \mathrm{Y}$ is said to be:

(i) g-continuous [4] if $f^{-1}(V)$ is g-closed in $\mathrm{X}$ for each closed set $\mathrm{V}$ of $\mathrm{Y}$.

(ii) rg-continuous [13] if $f^{-1}(V)$ is rg-closed in $\mathrm{X}$ for each closed set $\mathrm{V}$ of $\mathrm{Y}$.

(iii) rg-irresolute [13] if $f^{-1}(V)$ is rg-open in $\mathrm{X}$ for each rg-open set $\mathrm{V}$ of $\mathrm{Y}$.

(iv) gp-continuous [2] if $f^{-1}(V)$ is gp-closed in $\mathrm{X}$ for each closed set $\mathrm{V}$ of $\mathrm{Y}$.

(v) gp-irresolute [2] if $f^{-1}(V)$ is gp-open in $\mathrm{X}$ for each gp-open set $\mathrm{V}$ of $\mathrm{Y}$.

Definition 2.7: A topological space $X$ is said to be:

(i) p-regular [5] if for each closed set $\mathrm{A}$ and each point $\mathrm{x} \in \mathrm{X}$-AthereexistpreopensetsU, $V$ such that $\mathrm{x} \varepsilon \mathrm{U}, \mathrm{A} \subset \mathrm{V}$ and $\mathrm{U} \cap \mathrm{V}=\varnothing$.

(ii) g-regular [10] if for each closed set $A$ and each point $\mathrm{x} \in \mathrm{X}-\mathrm{A}$ there exist generalized open sets $\mathrm{U}, \mathrm{V}$ such that $\mathrm{x} \in \mathrm{U}$, $\mathrm{A} \subset \mathrm{V}$ and $\mathrm{U} \cap \mathrm{V}=\Phi$.

(iii) pre-connected [14] if $\mathrm{X}$ cannot be written as disjoint union of two non- empty preopen sets.

(iv) rg-connected [1] if $\mathrm{X}$ cannot be written as disjoint union of two non-empty rg-open sets.

\section{Properties of pre-Rg-continuous functions}

In this section we define the following. 
Definition 3.1: A function $\mathrm{f}: \mathrm{X} \rightarrow \mathrm{Y}$ is called pre-Rg-continuous if the inverse image of each $\operatorname{rg}$-open set of $\mathrm{Y}$ is preopen in X.

We have the following implications:

(i) Every strongly rg-continuous function is pre- $\mathrm{Rg}$-continuous.

(ii) Every pre-Rg-continuous function is almost precontinuous.

We prove the following.

Theorem 3.2: Let $\mathrm{f}: \mathrm{X} \rightarrow \mathrm{Y}$ be a single valued function, where $\mathrm{X}$ and $\mathrm{Y}$ are topological spaces, then the following are equivalent

(i) The function $\mathrm{f}$ is pre- $\mathrm{Rg}$-continuous.

(ii) For each point $x \in X$ and each rg-open set $\mathrm{V}$ in $\mathrm{Y}$ with $f(x) \in V$, there is a preopen set $\mathrm{U}$ in $\mathrm{X}$ such that $x \in U, f(U) \subset$ V.

Proof : (i) $\Rightarrow$ (ii):

Let $f(x) \in V$ and $V \subset Y$ an rg-open set then $x \in f^{-1}(V) \in P O(X)$ as $f$ is pre-Rg-continuous. Let $U=f^{-1}(V)$, then $\mathrm{x} \in U$ and $f(U) \subset V$. Conversely, Let $\mathrm{V}$ be rg-open in $\mathrm{Y}$ and $x \in f^{-1}(V)$ then $f(x) \subset V$, there exists a $\mathrm{Up} \in P 0(X)$ such that $\mathrm{p} \in \mathrm{Up}$ and $\mathrm{f}(\mathrm{Up}) \subset V$. Then $\mathrm{x} \in \mathrm{Ux} \subset f^{-1}(V)$ and $f^{-1}(V)=\mathrm{UUx} \in \mathrm{PO}(\mathrm{X})$. This implies $\mathrm{f}$ is pre-Rg-continuous.

We define the following.

Definition 3.3 :A function $\mathrm{f}: \mathrm{X} \rightarrow \mathrm{Y}$ is called gp-rg-continuous if the inverse image of each rg- open subset of $\mathrm{Y}$ is gpopen in X.

Clearly, Every pre-rg-continuous function is gp-rg-continuous function.

Theorem 3.4: Let $\mathrm{f}: \mathrm{X} \rightarrow \mathrm{Y}$ be pre-Rg-continuous then $\mathrm{f}$ is gp-rg-continuous.

Proof:Let $V \subset Y$ berg-open. Then $f^{-1}(V)$ is preopen in $\mathrm{X}$, sincef is pre-Rg-continuous. Since every preopen set is gp-open set, then $f^{-1}(V)$ is gp-open set in $\mathrm{X}$. Thus, $\mathrm{f}$ is gp-rg-continuous.

We define the following.

Definition 3.5: A function $\mathrm{f}: \mathrm{X} \rightarrow \mathrm{Y}$ is called gp-gpr-continuous if the inverse image of each gpr-open subset of $\mathrm{Y}$ is gp-open in X.

Clearly, Everygp-gpr-continuous function is gpr-irresolute.

Definition 3.6: A function $\mathrm{f}: \mathrm{X} \rightarrow \mathrm{Y}$ is called pre-strongly-gpr-continuous if the inverse image of each gpr-open set of $\mathrm{Y}$ is preopen in $\mathrm{X}$.

Note 3.7:

(i) Every strongly rg-continuous function is pre-Rg-continuous.

(ii) Every pre-Rg-continuous function is almost precontinuous.

Theorem 3.8: Let $\mathrm{f}: \mathrm{X} \rightarrow \mathrm{Y}$ be a function. Then the following are equivalent.

(i) $\mathrm{f}$ is pre-Rg-continuous.

(ii) The inverse image of each rg-open set in $\mathrm{Y}$ is preopen in $\mathrm{X}$.

(iii) The inverse image of each $\mathrm{rg}$-closed set in $\mathrm{Y}$ is preclosed in $\mathrm{X}$.

Proof :(i) $\Rightarrow$ (ii) :

Let $G$ be any rg-openset of $Y$. Then, Y-Gisrg-closed in $Y$. By the assumption of $(i), f^{-1}(Y-G)$ is preclosed set in $X$. But $f^{-1}(Y-G)=X-f^{-1}(G)$ which implies that $X-f^{-1}(G)$ is preclosed in $X$. Therefore $f^{-1}(G)$ is preopen in $X$.

(ii) $\Rightarrow$ (iii) : Obvious.

(iii) $\Rightarrow$ (i) : Obvious.

Theorem 3.9: Let $\mathrm{f}: \mathrm{X} \rightarrow \mathrm{Y}$ is pre-Rg-continuous surjection and $\mathrm{X}$ is preconnected then $\mathrm{Y}$ is $\operatorname{rg}$-connected.

Proof: Suppose $\mathrm{Y}$ is not rg-connected. Let $\mathrm{Y}=\mathrm{A} \cup \mathrm{B}$, where A and B are disjoint non-empty rg-open sets in $\mathrm{Y}$. Since $\mathrm{f}$ is pre-Rg-continuous and onto, $X=f^{-1}(A) \cup f^{-1}(B)$, where $f^{-1}(A)$ and $f^{-1}(B)$ are disjoint non-empty and preopen sets in $\mathrm{X}$. This contradicts the fact that $\mathrm{X}$ is preconnected. Hence $\mathrm{Y}$ is rg-connected.

Theorem 3.10: Let $\mathrm{f}: \mathrm{X} \rightarrow \mathrm{Y}$ is gp-rg-continuous surjection and $\mathrm{X}$ is gp-connected then $\mathrm{Y}$ is $\operatorname{rg}$-connected.

Proof: Suppose $\mathrm{Y}$ is not $\mathrm{rg}$-connected. Let $\mathrm{Y}=\mathrm{AUB}$, where A and B are disjoint non-empty rg-open sets in $\mathrm{Y}$. Since $\mathrm{f}$ is gp-rg-continuous and onto, $X=f^{-1}(A) \cup f^{-1}(B)$, where $f^{-1}(A)$ and $f^{-1}(B)$ are disjoint non-empty and gp-open sets in $\mathrm{X}$. This contradicts the fact that $\mathrm{X}$ is gp-connected. Hence $\mathrm{Y}$ is rg-connected. 
Theorem 3.11: Let $\mathrm{f}: \mathrm{X} \rightarrow \mathrm{Y}$ is pre-strongly-gpr-continuous surjection and $\mathrm{X}$ is preconnected then $\mathrm{Y}$ is gpr-connected. Proof: Suppose $\mathrm{Y}$ is not gpr-connected. Let $\mathrm{Y}=\mathrm{AUB}$, where $\mathrm{A}$ and $\mathrm{B}$ are disjoint non-empty gpr-open sets in $\mathrm{Y}$. Since $\mathrm{f}$ is pre-strongly-gpr-continuous and Onto, $X=f^{-1}(A) \cup f^{-1}(B)$, where $f^{-1}(A)$ and $f^{-1}(B)$ are disjoint non-empty and preopen sets in $\mathrm{X}$. This contradicts the fact that $\mathrm{Y}$ is preconnected. Hence $\mathrm{Y}$ is gpr-connected.

Theorem 3.12: Let $\mathrm{f}: \mathrm{X} \rightarrow \mathrm{Y}$ is strongly $\mathrm{rg}$-continuous surjection and $\mathrm{X}$ is connected then $\mathrm{Y}$ is $\mathrm{rg}$-connected.

Proof: Suppose $\mathrm{Y}$ is not rg-connected. Let $\mathrm{Y}=\mathrm{A} \cup \mathrm{B}$, where $\mathrm{A}$ and $\mathrm{B}$ are disjoint non-empty rg-open sets in $\mathrm{Y}$. Since $\mathrm{f}$ is strongly-rg-continuous and Onto, $X=f^{-1}(A) \cup f^{-1}(B)$, where $f^{-1}(A)$ and $f^{-1}(B)$ are disjoint non-empty and open sets in $\mathrm{X}$. This contradicts the fact that $\mathrm{X}$ is connected. Hence $\mathrm{Y}$ is rg-connected.

We define the following.

Definition 3.13: A function $\mathrm{f}: \mathrm{X} \rightarrow \mathrm{Y}$ is said to be contra-pre-Rg-continuous if the inverse image of each rg-open set of $\mathrm{Y}$ is preclosed in $\mathrm{X}$.

Definition 3.14: A function $\mathrm{f}: \mathrm{X} \rightarrow \mathrm{Y}$ is said to be contra strongly-rg-continuous if the inverse image of each rg-open set of $\mathrm{Y}$ is closed in $\mathrm{X}$.

Since every open set is rg-open set and every closed set is preclosed set and hence we have the following implications.

(i) Every contra strongly rg-continuous function is contra-pre-Rg-continuous.

(ii) Every contra-pre- Rg-continuous function is contra-precontinuous.

Theorem 3.15 : The following are equivalent for a function $\mathrm{f}: \mathrm{X} \rightarrow \mathrm{Y}$ :

(i) $\mathrm{f}$ is contra-pre- $\mathrm{Rg}$-continuous.

(ii) For each rg-closed subset $\mathrm{F}$ of $\mathrm{Y}, f^{-1}(F) \in P O(X)$,

(iii)For each $\mathrm{x} \in X$ and $\operatorname{rg}$-closed subset of $\mathrm{Y}$ containing $\mathrm{f}(\mathrm{x})$, there exists preopen set $\mathrm{U}$ in $\mathrm{X}$ containing point $\mathrm{x}$ such that $\mathrm{f}(\mathrm{U}) \subset \mathrm{F}$.

Proof : (i) $\Rightarrow$ (ii) :

Let $\mathrm{F}$ be any rg-closed subset of $Y$. Then, $Y$-F be any rg-open subset of $Y$. Since by $(i), f^{-1}(Y-F)=X-f^{-1}(F)$ is preclosed set in $\mathrm{X}$. Hence, $f^{-1}(F)$ is is preopen set in $\mathrm{X}$. Thus, (ii) holds.

(ii) $\Rightarrow$ (i) : Obvious.

(ii) $\Rightarrow$ (iii) : Obvious.

(iii) $\Rightarrow$ (ii) :

Let $\mathrm{F}$ be any rg-closed subset of $\mathrm{Y}$ and $\mathrm{x} \in \mathrm{f}^{-1}(F)$. Then $\mathrm{f}(\mathrm{x}) 000 \in F$ and there exists a preopen set $\mathrm{U}_{\mathrm{X}}$ in $\mathrm{X}$ containing point x such that $f\left(\mathbf{U}_{\mathbf{X}}\right) \subset \mathrm{F}$. Therefore, we obtain $f^{-1}(F)=U\left\{\mathbf{U}_{\mathbf{X}} \backslash \mathbf{x} \in f^{-1}(F)\right\} \in P O(X)$. This shows that (ii) holds. We define the following.

Definition 3.16 : A topological space $X$ is said to be pre-rg-regular if for each rg-closed set $\mathrm{A}$ and each point $\mathrm{x} \in X-A$ there exists preopen sets $\mathrm{U}, \mathrm{V}$ such that $\mathrm{x} \in U, A \subset V$ and $U \cap V=\Phi$.

Definition 3.17 : A topological space $X$ is said to be pre-g-regular if for each g-closed set A and each point $\mathrm{x} \in X-A$ there exists preopen sets $\mathrm{U}, \mathrm{V}$ such that $\mathrm{x} \in U, A \subset V$ and $U \cap V=\Phi$.

Since every closed set is g-closed set and every g-closed set is rg-closed set and every closed set is rg-closed set. Thus we have the following implications.

(i) Every pre- Rg-regular space is p-regular.

(ii) Every pre- g-regular space is p-regular.

(iii)Every pre- Rg-regular space is pre-g-regular.

We state the following.

Theorem 3.18: For a topological space $X$ the following hold:

(i) $\mathrm{X}$ is a pre-rg-regular.

(ii) For each $\mathrm{x} \in X$ and each rg-open set $\mathrm{U}$ of $\mathrm{X}$ containing $\mathrm{x}$, there exists $\mathrm{V} \in P O(X)$ such that $\mathrm{x} \in V \subset p C(V) \subset U$

(iii) For each rg-closed set $\mathrm{F}$ of $\mathrm{X}, \cap\{p C l(V) \mid F \subset V \in P O(X)\}=F$.

Theorem 3.19: For a topological space $X$ the following hold:

(i) $\mathrm{X}$ is a pre- g-regular.

(ii) For each $\mathrm{x} \in X$ and each g-open set $\mathrm{U}$ of $\mathrm{X}$ containing $\mathrm{x}$, there exists $\mathrm{V} \in P O(X)$ such that $\mathrm{x} \in V \subset p C(V) \subset U$

(iii) For each g-closed set $\mathrm{F}$ of $\mathrm{X}, \cap\{p C(V) \mid F \subset V \in P O(X)\}=F$

Theorem 3.20: If a function $f: X \rightarrow Y$ is said to be contra-pre-rg-continuous and $Y$ is pre- rg- regular, then $f$ is pre- $r g$ continuous.

Proof : Let $x$ be any arbitrary point of $X$ and $V$ be an rg-open set of $Y$ containing $f(x)$. Since $Y$ is pre- rg-regular, there exists an pre-open set $\mathrm{w}$ in $\mathrm{Y}$ containing $\mathrm{f}(\mathrm{x})$ such that $\mathrm{pCl}(\mathrm{w}) \subset \mathrm{V}$. Since $\mathrm{f}$ is contra- pre-rg-continuous, so by Theorem 3.15 there exists preopen set $\mathrm{U}$ in $\mathrm{X}$ containing point $\mathrm{x}$ such that $\mathrm{f}(\mathrm{U}) \subset \mathrm{pCl}(\mathrm{w})$. Then $\mathrm{f}(\mathrm{U}) \subset \mathrm{pCl}(\mathrm{w}) \subset \mathrm{V}$. Hence $\mathrm{f}$ is pre- 
rg-continuous.

We define the following

Definition 3.21 : A function $\mathrm{f}: \mathrm{X} \rightarrow \mathrm{Y}$ is said to be perfectly pre-Rg-continuous if $f^{-1}(V)$ is pre-regular set in $\mathrm{X}$ for each rg-open set $\mathrm{V}$ in $\mathrm{Y}$.

Lemma 3.22 :Let $\mathrm{f}: \mathrm{X} \rightarrow \mathrm{Y}$ be a function. Then,

(i) If $\mathrm{f}$ is perfectly pre- $\mathrm{Rg}$-continuous, then $\mathrm{f}$ is pre-rg-continuous.

(ii) If $\mathrm{f}$ is perfectly pre- $\mathrm{Rg}$-continuous, then $\mathrm{f}$ is Contra pre-Rg-continuous.

Proof:

(i) Let $\mathrm{U}$ be an rg-open set in $\mathrm{Y}$, since $\mathrm{f}$ is perfectly pre-Rg-continuous, then $f^{-1}(U)$ is pre-regular in $\mathrm{X}$. But every preregular set is pre-open, then $f^{-1}(U)$ is pre-open in X.Hence $\mathrm{f}$ is perfectly pre- Rg-continuous.

(ii) Let U be an rg-open set in Y, Since f is perfectly pre-Rg-continuous, then $f^{-1}(U)$ is pre-regular in X. But every pre-regular set is pre-closed, then $f^{-1}(U)$ is preclosed in X.Hence $\mathrm{f}$ is Contra pre- Rg-continuous.

Theorem 3.23: Let $\mathrm{f}: \mathrm{X} \rightarrow \mathrm{Y}$ be a function. Then the following are equivalent.

(i) If $\mathrm{f}$ is perfectly pre-Rg-continuous.

(ii) The inverse image of every rg-open set in $\mathrm{Y}$ is both preopen and preclosed in $\mathrm{X}$.

(iii) The inverse image of every rg-closed set in $\mathrm{Y}$ is both preopen and preclosed in $\mathrm{X}$.

Proof :

(i) $\Rightarrow$ (ii) : Clearly from the definition.

(ii) $\Rightarrow$ (iii) :Let $\mathrm{F}$ be any rg-closed set in $\mathrm{Y}$. Then, (Y-F) is rg-open in Y. Hence by assumption

(iii) $f^{-1}(Y-F)$ is both pre-open and pre-closed in $\mathrm{X}$.

(iv) $\Rightarrow$ (i) : Let $\mathrm{G}$ be any rg-open set in $\mathrm{Y}$. Then, $(\mathrm{Y}-\mathrm{G})$ is rg-closed in $\mathrm{Y}$. Hence by the condition

(v) $f^{-1}(Y-G)$ is both pre-open and pre-closed in $\mathrm{X}$ which implies that $f^{-1}(G)$ is both preopen and preclosed in $\mathrm{X}$. Hence $\mathrm{f}$ is perfectly pre- $\mathrm{Rg}$-continuous.

Theorem 3.25 :Let $\mathrm{f}: \mathrm{X} \rightarrow \mathrm{Y}$ and $\mathrm{g}: \mathrm{X} \rightarrow \mathrm{Y}$ be two functions. Then

(i) gof is pre-Rg-continuous, if $g$ is gp-rg-continuous and $f$ is pre- $\mathrm{Rg}$-continuous.

(ii) gof is pre- $\mathrm{Rg}$-continuous, if $\mathrm{g}$ is gp-rg-continuous and $\mathrm{f}$ is pre-strongly-gp-continuous.

(iii)gof is pre-Rg-continuous, if $\mathrm{g}$ is strongly-rg-continuous and $\mathrm{f}$ is pre-continuous.

\section{Proof :}

i. $\quad$ Let $\mathrm{V}$ be $\arg$-open set in $\mathrm{Z}$. As $\mathrm{g}: \mathrm{Y} \rightarrow \mathrm{Z}$ is rg-irresolute, $g^{-1}(V)$ is rg-open in $\mathrm{Y}$. Again, fispre-Rg-continuous and $g^{-1}(V)$ is rg-open in $Y$. Then $f^{-1}\left(g^{-1}(V)\right)=(g \circ f)^{-1}(V)$ Preopen set in X. Therefore gof is pre-Rg-continuous.

ii. Let $\mathrm{V}$ be $\arg$-open set in $\mathrm{Z}$. As $\mathrm{g}: \mathrm{Y} \rightarrow \mathrm{Z}$ is gp-rg-continuous, $g^{-1}(V)$ is gp-open in $\mathrm{Y}$. Again, $\mathrm{f}$ is pre-strongly-gpcontinuous and $g^{-1}(V)$ is gp-open in Y. Then $f^{-1}\left(g^{-1}(V)\right)=(g \circ f)^{-1}(V)$ is Preopen set in X. Therefore gof is pre-Rgcontinuous.

iii. Let $\mathrm{V}$ be $\arg$-open set in $\mathrm{Z}$. As $\mathrm{g}: \mathrm{Y} \rightarrow \mathrm{Z}$ is strongly-rg-continuous, $g^{-1}(V)$ is open in $\mathrm{Y}$. Again, $\mathrm{f}$ is pre-continuous and $g^{-1}(V)$ is open in Y. Then $f^{-1}\left(g^{-1}(V)\right)=(g \circ f)^{-1}(V)$ Preopen set in X. Therefore gof is pre-Rg-continuous.

Theorem 3.25 : Let $\mathrm{f}: \mathrm{X} \rightarrow \mathrm{Y}$ and $\mathrm{g}: \mathrm{X} \rightarrow \mathrm{Y}$ be two functions. Then

(i) gof is precontinuous, if $\mathrm{f}$ is pre- $\mathrm{Rg}$-continuous and $\mathrm{g}$ is $\mathrm{rg}$-continuous.

(ii) gof is precontinuous, if $\mathrm{f}$ is pre-strongly-rg-continuous and $\mathrm{g}$ is $\mathrm{rg}$-continuous.

(iii)gof is continuous, if $\mathrm{f}$ is strongly-rg-continuous and $\mathrm{g}$ is $\mathrm{rg}$-continuous.

Proof :

i. Let $\mathrm{V} \subset \mathrm{Z}$ be an arbitrary open set. Since $\mathrm{g}$ is rg-continuous, $g^{-1}(V)$ is rg-open set in $\mathrm{Y}$. Again, as $g^{-1}(V)$ is $\mathrm{rg}^{-}$ open set in $\mathrm{Y}$ and $\mathrm{f}$ is pre-Rg-continuous function, $f^{-1}\left(g^{-1}(V)\right)=(g \circ f)^{-1}(V)$ is preopen set in $\mathrm{X}$. This shows that gof is precontinuous function.

ii. Let $\mathrm{V} \subset \mathrm{Z}$ be an arbitrary open set. Since $\mathrm{g}$ is rg-continuous function, $g^{-1}(V)$ isrg- open set in Y. Again, as $g^{-1}(V)$ is rg-open set in $\mathrm{Y}$ and $\mathrm{f}$ is pre-strongly-rg- continuous function, $f^{-1}\left(g^{-1}(V)\right)=(g \circ f)^{-1}(V)$ is preopen set in X. This shows that gof is precontinuous function.

iii. Let $\mathrm{V} \subset \mathrm{Z}$ be an arbitrary open set. Since $\mathrm{g}$ is rg-continuous function, $g^{-1}(V)$ is rg- open set in $\mathrm{Y}$. Again, as $g^{-1}(V)$ is rg-open set in $\mathrm{Y}$ and $\mathrm{f}$ is strongly-rg-continuous function, $f^{-1}\left(g^{-1}(V)\right)=(g \circ f)^{-1}(V)$ is open set in X. This shows that gof is continuous function.

Lemma 3.26 : If $\mathrm{f}: \mathrm{X} \rightarrow \mathrm{Y}$ is strongly preopen surjective and $\mathrm{g}: \mathrm{Y} \rightarrow \mathrm{Z}$ is a function such that gof $: \mathrm{X} \rightarrow \mathrm{Z}$ is pre-Rgcontinuous then $\mathrm{g}$ is strongly -rg-continuous.

Proof : Let V be an arbitrary open set in Z. Since gof is pre-Rg-continuous, $(g \circ f)^{-1}(U)=f^{-1}\left(g^{-1}(U)\right)$ is preopen in $X$. Since $\mathrm{f}$ is strongly preopen surjective, $\mathrm{f}\left(f^{-1}\left(g^{-1}(U)\right)\right)=g^{-1}(U)$ is open in Y. Therefore $\mathrm{g}$ is strongly-rg-continuous. 
Lemma 3.28 $:$ If $\mathrm{f}: \mathrm{X} \rightarrow \mathrm{Y}$ and $\mathrm{g}: \mathrm{Y} \rightarrow \mathrm{Z}$ be two functions. If $\mathrm{f}$ is precontinuous, $\alpha$-open and $\mathrm{g}$ is pre- $\mathrm{Rg}$-continuous, then gof $: \mathrm{X} \rightarrow \mathrm{Z}$ is pre-Rg-continuous .

Proof: Let $\mathrm{V}$ be an arbitrary rg-open subset of $\mathrm{Z}$. Then $g^{-1}(V)$ is preopen set in $\mathrm{Y}$, since $\mathrm{g}$ is pre- $\mathrm{Rg}$-continuous. As, fis precontinuous and $\alpha$-open and $g^{-1}(V)$ is preopen, $f^{-1}\left(g^{-1}(V)\right)=(g \circ f)^{-1}(V)$ is preopen in $\mathrm{X}$. This shows that gof is pre-Rgcontinuous.

Lemma 3.28: If $\mathrm{f}: \mathrm{X} \rightarrow \mathrm{Y}$ is surjective $\mathrm{M}$-preopen and $\mathrm{g}: \mathrm{Y} \rightarrow \mathrm{Z}$ is a function such that gof $: \mathrm{X} \rightarrow \mathrm{Z}$ is pre-Rg-continuous then $\mathrm{g}$ is pre- $\mathrm{Rg}$-continuous.

Proof : Let U be an rg-open set in Z. Since gof is pre-Rg-continuous, $(g \circ f)^{-1}(U)=f^{-1}\left(g^{-1}(U)\right)$ is preopen in X. Since $f$ is surjective M-preopen, $\operatorname{if}\left(f^{-1}\left(g^{-1}(U)\right)\right)=g^{-1}(U)$ is preopen in Y. Therefore, $g$ is pre- $\operatorname{Rg}$-continuous.

Theorem 3.29: If $\mathrm{f}: \mathrm{X} \rightarrow \mathrm{Y}$ is rg-irresolute and $\mathrm{g}: \mathrm{Y} \rightarrow \mathrm{Z}$ be g-rg-continuous, then gof $: \mathrm{X} \rightarrow \mathrm{Z}$ is g-rg-continuous. Proof: Let $\mathrm{V}$ be an arbitrary g-open subset of $\mathrm{Z}$. Then $g^{-1}(V)$ is rg-open set $\mathrm{n}$ Y, Since $\mathrm{g}$ is g- rg-continuous. As, $\mathrm{f}$ is rgirresolute and $g^{-1}(V)$ is rg-open, $f^{-1}\left(g^{-1}(V)\right)=(g \circ f)^{-1}(V)$ is rg- open in X. This shoes that gof is g-rg-continuous.

Theorem 3.30 : If $\mathrm{f}: \mathrm{X} \rightarrow \mathrm{Y}$ be strongly g-continuous with $\mathrm{Y}$ be Trgspace and $\mathrm{g}: \mathrm{Y} \rightarrow \mathrm{Z}$ be $\mathrm{g}$ - rg-continuous, then gof $: X \rightarrow Z$ is strongly g-continuous.

Proof : Let $\mathrm{V} \subset \mathrm{Z}$ be an arbitrary g-open set. Since $\mathrm{g}$ is g-rg-continuous, then $g^{-1}(V)$ is rg-open set $\mathrm{n} \mathrm{Y}$. Given that $\mathrm{Y}$ is Trgspace,$g^{-1}(V)$ is g-open in $\mathrm{Y}$ and $\mathrm{f}$ is strongly g-continuous, $f^{-1}\left(g^{-1}(V)\right)=(g \circ f)^{-1}(V)$ isopeninX. Thisshowsthatgof is stronglyg-continuous.

Theorem 3.31 : If $\mathrm{f}: \mathrm{X} \rightarrow \mathrm{Y}$ be pre-Rg-continuous and $\mathrm{g}: \mathrm{Y} \rightarrow \mathrm{Z}$ be pre-rg-continuous, then gof $: \mathrm{X} \rightarrow \mathrm{Z}$ is almost precontinuous.

Proof : Let V be an arbitrary regular-open set of $\mathrm{Z}$. Since $\mathrm{g}$ is pre-rg-continuous, then $g^{-1}(V)$ is rg-open set $\mathrm{n} \mathrm{Y}$, As $\mathrm{f}$ is pre-Rg-continuous function and $g^{-1}(V)$ is rg-open in Y. Then, $f^{-1}\left(g^{-1}(V)\right)=(g \circ f)^{-1}(V)$ ispreopen in X. This shows that gof is almost precontinuous.

\section{References}

[1].I. Arokarani, K. Balchandran, 'On Regular Generalized Continuous Maps in Topological Spaces', Kyungpook Math.J.37 (1997), 305-314.

[2].I. Arokarani, K.Balchandran and J. Dontchev, Some Characterizations of gp-irresolute And gp-continuous maps between topological spaces Mem. Fac. Sci. Kochi Univ. (Math.) 20 (1999), 93-104.

[3].S. P. Arya and R. Gupta (1974), 'On Strongly Continuous Functions', Kyungpook Math. J., 14 :131-143.

[4].K.Balachndran, P. Sundaram and H. Maki, 'On generalized Continuous maps in Topological Spaces', Mem. Fac. Sci. Kochi. Univ. (Math) 12 (1991),5-13.

[5].S.N.El - Deeb, I. A. Hasanein, A. S. Mashhour and T. Noiri, “ On p-regular Spaces”, Bull. Math. Soc.Sci. Math. R.S.Roumanie (N.S), 27 (75), (1983), 311-315.

[6].Y.GNANAMBAL, 'On Generalized preregular closed set in Topological Spaces', Indian. J. Pure. Appl. Math., 28(3) : 351-360, March 1997.

[7].N.Levine, Generalized Closed Sets in Topology. Rend. Circ. Mat. Palermo. 19(2) (1970), 89-96.

[8].A. S. Mashhour, M.E.Abd El-Monsef and S.N.El-Deeb, On Precontinuous and weak precontinuous mappings, Proc. Math. Phys. Soc. Egypt, 53(1982) 47-53.

[9].A. S. Mashhour, M. E. Abd El-Monsef and I.A.Hasanein "On pretopological Spaces”, Bull. Math. Soc. Sci. Math. R.S. Roumanie, 28(76) (1984), 39-45.

[10]. B. M. Munshi, 'Separation Axioms', Acta Crencia Indica 12(1986), 140-145.

[11]. Govindappa Navalagi, ' $\alpha$-Neighborhoods in Topological Spaces', pacific- Asian Journal of Mathematics, Volume 3, No.1-2. January - December 2009.

[12]. T.Noiri, H. Maki and J.Umehara, 'Generalized preclosed Functions' Mem. Fac. Sci. Kochi. Univ. (Math.) 19(1998), 13-20.

[13]. N.Palniappan and K. Chandrashekar Rao, 'Regular Generalized Closed Sets', Kyungpook Mathematical Journal, Vol.33, No.2, 211-219, December 1993.

[14]. V. Popa, "Properties of H-almost continuous Functions", Bull. Math. Soc. Sci. Math.

RSR. Tome 31(79), no.2, (1987), 163-168. 